\title{
Europäischer und Deutscher Qualifikationsrahmen
}

\author{
HERMANN NEHLS
}

Die Europäische Union (EU) hat im Jahr 2000 das Ziel vorgegeben, die Union zum „wettbewerbsfähigsten und dynamischsten wissensbasierten Wirtschaftsraum in der Welt" zu machen, die sogenannte Lissabon-Strategie. Die Lissabon-Strategie beinhaltet eine explizite strategische Ausrichtung der Berufsbildungspolitik auf wirtschafts- und wettbewerbspolitische Ziele. Von den Lissabon-Zielen wurden insgesamt nur wenige erreicht, doch die vorläufige Bilanz für den Bereich der Berufsbildung sieht anders aus: Es gab und gibt eine lebhafte Debatte zu den Instrumenten, mit denen die Europäisierung der beruflichen Bildung vorangetrieben werden sollte. Alle Mitgliedstaaten erarbeiten nationale Qualifikationsrahmen. Sie beteiligen sich an der Umsetzung der Empfehlung des Europäischen Rahmens für Qualitätssicherung in der Beruflichen Bildung (EQARF), am Europäischen Leistungspunktesystem für berufliche Bildung (ECVET), und nicht zuletzt der Validierung nonformal und informell erworbener Kompetenzen.

Die Mitgliedstaaten der Europäischen Union sollen ihre eigenen nationalen Qualifikationsrahmen oder anderweitige Einordnungssysteme mit dem Europäischen Qualifikationsrahmen (EQR) ${ }^{1}$ verknüpfen. Alle individuellen Qualifikationsbescheinigungen sollen dann einen Verweis auf das zutreffende EQR-Niveau enthalten. Das haben die EU-Mitglieder verbindlich zugesagt. Doch es ist schwierig, die unterschiedlichen Ausbildungssysteme der EU zu vergleichen. Die duale Berufsausbildung der Bundesrepublik Deutschland gibt es vergleichbar nur in Dänemark, Österreich und der Schweiz (die nicht Mitglied der EU ist). Der EQR soll hier helfen.

Der EQR ist Teil der EU-Strategie, einen europäischen Arbeitsmarkt und Bildungsraum mit mehr Transparenz und Mobilität zu schaffen. Dabei dient der EQR als Instrument zur Förderung des lebenslangen Lernens. Als europäischer Metarahmen, der die verschiedenen nationalen Qualifikationsrahmen miteinander verknüpft, führt er die Bildungssysteme näher aneinander heran.

Der Deutsche Gewerkschaftsbund (DGB) unterstreicht die Zielsetzung europäischer Bildungspolitik, bisher voneinander abgeschottete Bildungssysteme aufeinander $\mathrm{zu}$ beziehen und die Durchlässigkeit im Bildungssystem zu erhöhen. Gleichwertigkeit von allgemeiner und beruflicher Bildung, Transparenz und Durchlässigkeit in der Bildung, Anerkennung von Berufserfah- rung und lebensbegleitendes Lernen sind seit Jahren gewerkschaftliche Forderungen. Die Gewerkschaften kritisieren schon seit geraumer Zeit, dass die Durchlässigkeit zwischen den unterschiedlichen Bildungseinrichtungen und -systemen unzureichend ist.

\section{Entwicklung des Deutschen Qualifikations- rahmens}

Im Mai 2013 wurde der Deutsche Qualifikationsrahmen (DQR) als gemeinsamer Beschluss mit einem Leitfaden und ersten Zuordnungen zu den Niveaus veröffentlicht. ${ }^{2}$ Die DQR-Matrix unterscheidet sich in ihrer Architektur und Terminologie deutlich vom EQR-Ansatz. Während der EQR auf drei Säulen beruht (Wissen, Fertigkeiten und Kompetenz), kommt die zentrale Stellung der Handlungskompetenz im DQR durch die Beschreibung der Anforderungssituation des jeweiligen Niveaus und einer Viersäulenstruktur (Wissen, Fertigkeiten, Sozialkompetenz und Selbstständigkeit) besser zum Ausdruck. Dem EQR liegt zudem ein eingeschränktes Kompetenzverständnis zugrunde, weil Kompetenz nur mit der Übernahme von Verantwortung und Selbstständigkeit beschrieben wird.

Die Vier-Säulen-Struktur war im Arbeitskreis DQR, in dem Repräsentanten aller Bildungsbereiche einschließlich der Arbeitgeberseite und der Gewerkschaften seit 2006 an der Entwicklung des DQR arbeiten, lange Zeit umstritten. Während die Vertreterinnen und Vertreter der Ministerien, der Kultusministerkonferenz (KMK) und der Hochschulseite eine engere Ausrichtung am EQR, also eine Drei-Säulen-Struktur favorisierten, konnten sich am Ende die Arbeitgeberseite und die Gewerkschaften mit der jetzt veröffentlichten Vier-Säulen-Struktur durchsetzen. Hierdurch sollte Handlungskompetenz besser abgebildet werden können. Die Zuordnung von beruflichen Qualifikationen auf den höheren Niveaus ist so viel besser möglich, weil berufliches Wissen stärker Berücksichtigung findet.

1 Vgl. http: //ec.europa.eu/education/lifelong-learning-policy/doc44_de.htm.

2 Vgl. www.deutscherqualifikationsrahmen.de. 


\section{Zur Kompetenzdebatte}

Auf den ersten Blick schien zwischen den in Deutschland beteiligten Akteuren die größte Übereinstimmung im Hinblick auf die Ziele eines DQR vorzuliegen. So hatte beispielsweise die Arbeitsgruppe des Hauptausschusses des Bundesinstituts für Berufsbildung (BIBB) zur Erarbeitung eines Deutschen Qualifikationsrahmens, an der Vertreter der Arbeitgeberseite, der Ministerien und der Gewerkschaften teilnahmen, einen Konsens darüber erzielt, welche Ziele verfolgt werden sollten. Die Ausrichtung entsprach im Wesentlichen der des EQR. Die Spitzenverbände der Wirtschaft ergänzten den Zielkanon allerdings um den Hinweis, dass ein EQR nur dann gelingen könne, „wenn er am Bedarf der Wirtschaft und am Nutzen für die Unternehmen ausgerichtet ist“. ${ }^{3}$ Ähnlich äußerten sich Branchenverbände der Metall- und Elektroindustrie: Entscheidend seien die Einordnung von Kompetenzen und beruflicher Handlungsfähigkeit sowie die Orientierung an den qualifikatorischen Anforderungen des Beschäftigungssystems. ${ }^{4}$ Dies ist eine einseitige utilitaristische Ausrichtung; die gesellschaftliche Dimension von Bildung, auch beruflicher Bildung, wird ausgeblendet.

Die Gewerkschaften haben sich dagegen für einen Kompetenzbegriff starkgemacht, der berufliche, personale und gesellschaftliche Dimensionen beinhaltet. Er zielt auf berufliche Handlungsfähigkeit und persönliche Entwicklung unter Einschluss von Planungs- und Entscheidungsfähigkeit ab. ${ }^{5}$ Bezugspunkte sind: ganzheitliche Arbeitsaufgaben, die Anforderungen des Arbeitsmarktes unter dem Aspekt langfristiger Verwertbarkeit der Qualifikationen, die individuelle Kompetenzentwicklung, Mitwirkung an betrieblichen und gesellschaftlich-sozialen Prozessen und reflexive Handlungsfähigkeit.

Die Arbeitsgruppe des Hauptausschusses des BIBB hat sich nach intensiver Diskussion auf eine Definition verständigt, was unter Handlungskompetenz zu verstehen ist. Diese lautet: „Handlungskompetenz wird als Einheit von Fach-, Sozial- und Human- bzw. personaler Kompetenz definiert. Sie dient der Bewältigung unterschiedlich komplexer Anforderungen in Arbeits- und Lernsituationen. Sie versetzt damit, basierend auf Wissen und Erfahrung, Menschen in die Lage, gefundene Lösungen zu bewerten und die eigene Handlungsfähigkeit weiterzuentwickeln. Eine umfassende Handlungskompetenz ist unabdingbare Voraussetzung für Beruflichkeit, nachhaltige Beschäftigungsfähigkeit und fördert die gesellschaftliche Teilhabe. “6 Diese Definition entspricht derjenigen der Kultusministerkonferenz (KMK). ${ }^{7}$ Länder, die die Terminologie und Systematik des EQR übernommen haben, schließen beruflich Qualifizierte faktisch von den Niveaus 6, 7 und 8 aus. Diese Niveaus sind vornehmlich Absolventinnen und Absolventen der Hochschule (Bachelor, Master und Doktor) vorbehalten. Dies widerspricht dem Anliegen des EQR, Transparenz und Mobilität durch eine bildungsbereichsübergreifende Zuordnung für allgemeine, hochschulische und berufliche Bildung auf allen Niveaus zu fördern. Der EQR wird so zur Mogelpackung.

\section{Outcome-Orientierung}

Der EQR gilt für alle Arten allgemeiner und beruflicher Bildung und Qualifikationen, von der Schul- und Berufsbildung bis zur Hochschulbildung. Dies bedeutet eine Abkehr vom traditionellen Ansatz, bei dem Lerninputs wie Dauer einer Lernerfahrung oder Art der Einrichtung im Mittelpunkt stehen. Diese vorrangige Orientierung darauf, was jemand kann, und nicht, wo jemand gelernt hat (Outcome-Orientierung), grenzt sich eindeutig ab von einer Bildungstradition, in der Lerninhalte, Lernprozesse und Lernergebnisse curricular aufeinander abgestimmt und entsprechend beschrieben werden. Die reine Outcome-Orientierung berücksichtigt nicht, dass die Qualität von Lernergebnissen vor allem davon abhängt, wie vorhergehende Lernprozesse gestaltet werden.

Für die Gewerkschaften steht deshalb außer Frage, dass die Outcome-Orientierung eng mit einer Qualitätssicherung von Input und Prozess verknüpft werden muss. Gesellschaftlich normierte und standardisierte Lernwege, wie und soweit sie in Aus- und Weiterbildungsordnungen verankert sind, dürfen nicht durch beliebige marktorientierte Lernvorgaben, bei denen es völlig beliebig ist, wie und wo gelernt wird, ersetzt werden.

Input-, Prozess- und Outcome-Orientierung liegen der in Form von Ausbildungs- und Fortbildungsordnungen fixierten Beruflichkeit seit jeher zugrunde. Die mit
3 Brunner, S./Esser, F. H./Kloas, P.-W. (2006): Der Europäische Qualifikationsrahmen - Bewertung durch die Spitzenverbände der deutschen Wirtschaft, in: Berufsbildung in Wissenschaft und Praxis 35 (2), S. 14-17.

4 Bundesverband Informationswirtschaft, Telekommunikation und neue Medien/Gesamtverband der Arbeitgeberverbände der Metall- und Elektro-Industrie/Verband Deutscher Maschinen und Anlagenbau/Zentralverband Elektrotechnik und Elektronikindustrie (Hrsg.) (2007): Die Anforderungen des Beschäftigungssystems - Ein Beitrag zur Gestaltung des Deutschen Qualifikationsrahmens, Berlin/Frankfurt a. M.
5 Deutscher Gewerkschaftsbund (2008): Der Deutsche Qualifikationsrahmen (DQR). Chancen und Risiken aus gewerkschaftlicher Sicht, Berlin.

6 Hauptausschuss des Bundesinstituts für Berufsbildung, Arbeitsgruppe DOR/ECVET (2007): Leitlinien zur Gestaltung eines DQR, unveröffentlichtes Dokument. Siehe auch Ders. [o.J.]: Empfehlungen zur Erarbeitung eines deutschen Qualifikationsrahmens, www.bibb.de.

7 Kultusministerkonferenz (KMK) (2007): Handreichung für die Erarbeitung von Rahmenlehrplänen der Kultusministerkonferenz für den berufsbezogenen Unterricht in der 
ÜBERSICHT 1

\section{Die (bisher) zugeordneten formalen Qualifikationen im Deutschen Qualifikationsrahmen}

\begin{tabular}{|c|c|}
\hline Niveau & Qualifikationen \\
\hline 1 & $\begin{array}{l}\text { Berufsausbildungsvorbereitung } \\
\text { - Maßnahmen der Arbeitsagentur (BvB) } \\
\text { - Berufsvorbereitungsjahr (BVJ) }\end{array}$ \\
\hline 2 & $\begin{array}{l}\text { Berufsausbildungsvorbereitung } \\
\text { - Maßnahmen der Arbeitsagentur (BvB) } \\
\text { - Berufsvorbereitungsjahr (BVJ) } \\
\text { - Einstiegsqualifizierung (EQ) } \\
\text { Berufsfachschule (Berufliche Grundbildung) }\end{array}$ \\
\hline 3 & $\begin{array}{l}\text { Duale Berufsausbildung (2-jährige Ausbildungen) } \\
\text { Berufsfachschule (mittlerer Schulabschluss) }\end{array}$ \\
\hline 4 & $\begin{array}{l}\text { Duale Berufsausbildung (3- und } 31 / 2 \text {-jährige Ausbildungen) } \\
\text { Berufsfachschule (Assistentenberufe) } \\
\text { Berufsfachschule (vollqualifizierende Berufsausbildung) }\end{array}$ \\
\hline $5^{*}$ & $\begin{array}{l}\text { IT-Spezialist (Zertifizierter) } \\
\text { Servicetechniker (Geprüfter) }\end{array}$ \\
\hline 6 & $\begin{array}{l}\text { Bachelor } \\
\text { Fachkaufmann (Geprüfter) } \\
\text { Fachschule (Staatlich Geprüfter) } \\
\text { Fachwirt (Geprüfter) } \\
\text { Meister (Geprüfter) } \\
\text { Operativer Professional (IT) (Geprüfter) }\end{array}$ \\
\hline 7 & $\begin{array}{l}\text { Master } \\
\text { Strategischer Professional (IT) (Geprüfter) }\end{array}$ \\
\hline 8 & Promotion \\
\hline
\end{tabular}

* Weitere Qualifikationen der beruflichen Aufstiegsfortbildung werden nach dem im "Gemeinsamen Beschluss" beschriebenen Verfahren konsensual zugeordnet.

Quelle: Darstellung des Autors. nicht formalen und informellen Lernens fördern soll. In verschiedenen Ländern wird an Zertifizierungsmodi für solche Lernformen gearbeitet, um entsprechende Kompetenzen sichtbar zu machen. In einigen europäischen Ländern (Schweiz, Frankreich, Portugal, u. a.) $)^{9}$ gibt es Anerkennungsprozeduren, die auch bisher eher versteckte, beruflich relevante Kompetenzen transparent machen können.

Die Validierung nicht formalen und informellen Lernens hat in der Bundesrepublik Deutschland noch wenig Gewicht. Berufliche Qualifikationen werden fast ausschließlich über formelle Bildungsgänge erfasst. Qualifikationsnachweise beruhen weitgehend auf formalisierten Bildungsgängen und Prüfungen. Lernen, das sich außerhalb der formalisierten Bildung in offenen Kontexten vollzieht, wird nur in geringem Maße dokumentiert.

Seit Dezember 2012 liegt eine Empfehlung der Europäischen Kommission vor, wonach die Mitgliedstaaten bis 2018 Verfahren zur Validierung non-formal und informell erworbener Kompetenzen erarbeiten sollen. Die Entwicklung des Deutschen Qualifikationsrahmens bietet die Chance für eine systematische Einbindung der Anerkennung von non-formalem und informellem Lernen in das Qualifikationssystem. Notwendig ist ein formeller Rahmen, in dem die Anerkennung von non-formalem und informellem Lernen vollzogen wird. Informell erworbene Kompetenzen spielen vor allem im Bereich der beruflichen Weiterbildung eine erhebliche Rolle. Deren Anerkennung hängt vom Wohlwollen der Arbeitgeber ab, Betriebs- und Personalräte haben hier kaum Handlungsmöglichkeiten. Ein System der Validierung non-formal und informell erworbener Kompetenzen, das Information und Beratung, normierte Verfahren für die Erfassung, Bewertung und die Prüfung unter Beteiligung der Gewerkschaften einschließt, könnte neue Entwicklungsmöglichkeiten für die Beschäftigten bieten.

\section{Zuordnung zu den Niveaus des DQR} von Qualifikationen festgelegt worden, die auf die Outcome-Orientierung als die einzulösende Berufsfähigkeit zielen. Die Berufsfähigkeit des professionellen Handwerkers, Facharbeiters, Fachangestellten, Meisters und anderer Berufe bilden den Orientierungsrahmen für die Aus- und Weiterbildung der Betriebe und berufsbildenden Schulen. Dabei wird über berufsinhaltliche Grundlagen und die Qualitätsgestaltung des Qualifizierungsprozesses die Inputund Prozess-Orientierung gleichberechtigt einbezogen.

\section{Anerkennung non-formal und informell erworbener Kompetenzen}

Im Zusammenhang mit der Umsetzung der Lissabon-Strategie wird auch die Anerkennung informellen Lernens gefordert. ${ }^{8}$ Die Empfehlung zur Entwicklung eines Europäischen Qualifikationsrahmens beinhaltet, beider Beschreibung und Definition von Qualifikationen einen Ansatz zu verwenden, der auf Lernergebnissen beruht und der die Validierung
Bei der Zuordnung von Qualifikationsprofilen (Übersicht 1) standen vor allem die Frage des Verhältnisses von hochschulischen und beruflichen Aufstiegsfortbildungsgängen und die Frage des Verhältnisses von allgemein schulischer und beruflicher Bildung im Vordergrund.

Berufsschule und ihre Abstimmung mit Ausbildungsordnungen des Bundes für anerkannte Ausbildungsberufe, Bonn, www.kmk.org/doc/publ/handreich.pdf (letzter Zu-

8 Europäische Kommission (2008): Der Europäische Qualifikationsrahmen für lebenslanges Lernen (EQR), Luxemburg.

9 Dehnbostel, P./Seidel, S./Stamm-Riemer, I./Leykum, B. (2010): Einbeziehung von Ergebnissen informellen Lernens in den DQR - eine Kurzexpertise, Bonn/Hannover. griff: 07.02.2008), S. 10 
Der DQR enthält bildungsbereichsübergreifende Deskriptoren für die Zuordnung zu allen Niveaus. Dies schließt auch die in Europa heiß umkämpften Niveaus 6, 7 und 8 ein. Während diese Niveaus in den meisten Mitgliedstaaten von der Hochschule dominiert werden und Bachelor, Master und PhD hier ein Alleinstellungsmerkmal haben, sind im DQR die Voraussetzungen geschaffen worden, beruflich Qualifizierte auch den höchsten Niveaus zuzuordnen, ohne dass sie die Hochschule auch nur einen Tag von innen gesehen haben.

Im ersten Schritt wurden dem DQR Qualifikationen aus allen Bildungsbereichen zugeordnet, die sich durch besondere Arbeitsmarktrelevanz auszeichnen. Sie dienen als Anker- bzw. Referenzqualifikationen für die $\mathrm{Zu}-$ ordnung weiterer Qualifikationen zum DQR. Weitere Qualifikationen, insbesondere solche der beruflichen Weiterbildung, sind derzeit noch in der Beratung und werden nach dem im "Gemeinsamen Beschluss“ ${ }^{10}$ beschriebenen Verfahren konsensual zugeordnet. Die Zuordnungsliste wird entsprechend der Weiterentwicklung des DQR kontinuierlich erweitert.

Noch nicht abgeschlossen ist die Diskussion um das Verhältnis zwischen allgemein schulischen und beruflichen Bildungsgängen. Die KMK beharrt auf einer Zuordnung des „deutschen Abiturs“ auf Niveau 5, während die Berufe nach Berufsbildungsgesetz und Handwerksordnung differenziert den Niveaus 3 bis 5 zugeordnet werden sollen. Das Gros der Ausbildungsberufe würde dann unterhalb der allgemeinen Hochschulreife zugeordnet werden. Dies wird von Gewerkschaften und Arbeitgeberverbänden abgelehnt.

Mit der Zuordnung der Allgemeinen Hochschulreife auf Niveau 5 wäre eine differenzierte Zuordnung der Fachhochschulreife (auf Niveau 4) und der allgemeinen Hochschulreife (AHR) (auf Niveau 5) verbunden. Die Fachhochschulreife ist neben der allgemeinen Hochschulreife und der fachgebundenen Hochschulreife die dritte Form einer Hochschulzugangsberechtigung innerhalb des Schulsystems und ermöglicht - wie auch die anderen Formen der Hochschulzugangsberechtigung - den Abschluss eines Bachelors.

In konsensualer Abstimmung aller am DQR-Prozess Beteiligten ist es gelungen, Meister und Fachwirte ebenso wie Bachelor dem Niveau 6 zuzuordnen. Ab Niveau 6 beinhaltet die DQR-Matrix in der Kategorie Wissen eine Formulierung, die eine Zuordnung sowohl von hochschulisch (wissenschaftliche Grundlagen), wie auch von beruflich Qualifizierten (berufliches Wissen) möglich macht. Damit können verschiedene Qualifikationswege als gleichwertig, aber nicht gleichartig dargestellt und zugeordnet werden. Dies ist das Ergebnis langjähriger Aushandlungsprozesse zwischen den Gewerkschaften und Arbeitgeberverbänden, den Akteuren der Berufsbildung, des Bundesministeriums für Bildung und Forschung (BMBF), der KMK und der Hochschulrektorenkonferenz (HRK). Aktuell laufen noch Gespräche mit der HRK, um auch eine konsensuale Zuordnung beruflich Qualifizierter wie Betriebswirte nach Berufsbildungsgesetz und nach der Handwerksordnung zum Niveau $7 \mathrm{zu}$ erreichen. Ein Positivbeispiel: Der Strategische Professional IT wurde von allen Beteiligten als Ankerqualifikation angesehen, die aufbauend auf dem Spezialisten, Niveau 5, und dem Operativen Professional IT, Niveau 6 , dem Niveau 7 zugeordnet wird. Diese Fortbildungsqualifikation erfüllt alle Kriterien, die im Niveau 7 der DQR-Matrix beschrieben sind. Sie ist damit gleichwertig dem Masterabschluss zugeordnet.

\section{Fazit}

Der Deutsche Qualifikationsrahmen verbessert die Voraussetzungen für berufliche Entwicklungsmöglichkeiten für die Beschäftigten. Durch die bildungsbereichsübergreifende DQR-Matrix werden ab sofort beruflich Qualifizierte wie Meister und Fachwirte dem Niveau 6 zugeordnet, einem Niveau, das in anderen Mitgliedstaaten üblicherweise nur hochschulisch Qualifizierten vorbehalten ist. Beruflich Qualifizierte sind damit in der DQR-Logik auf Augenhöhe mit hochschulisch Qualifizierten. Das ist gesellschafts- und bildungspolitisch ein Durchbruch. Es verbessert das gesellschaftliche Ansehen und die Attraktivität der Berufsbildung. Bezeichnenderweise hat das bereits lohn- und gehaltspolitische Auswirkungen und ist nicht nur ein Wohlfühlprogramm für die Beschäftigten: In Tarifverträgen des Öffentlichen Dienstes wurde der Techniker mit Verweis auf seine Zuordnung zum Niveau 6 im DQR ein bis zwei Gehaltsstufen höher eingruppiert und ist damit dem Bachelor gleichgestellt worden.

Auch wenn es bezüglich der Zuordnung auch zu dem Niveau 7, dem auch Master zugeordnet werden, bereits eine grundsätzliche Verständigung für eine Zuordnung auch für beruflich Qualifizierte gibt, steht eine Einigung für konkrete berufliche Aufstiegsqualifikationen über den Strategischen Professional IT hinaus noch aus.

Eine weitere bildungspolitische Dynamik wird durch die Empfehlung an die Mitgliedstaaten ausgelöst, bis zum Jahr 2018 Verfahren zur Validierung non-formal und informell erworbener Kompetenzen zu entwickeln. Damit können für Deutschland Standards für non-formal und informell erworbene Kompetenzen entwickel werden, die auf tarifpolitischer und betrieblicher Ebene von Betriebs- und Personalräten genutzt werden können. Vor allem für den Bereich der beruflichen Weiterbildung kann damit Berufserfahrung, die nach der Berufsausbildung erworben wird, systematisiert und bewertet werden. Qualifikationen werden damit breiter erfasst, es zählen nicht nur formal erworbene Abschlüsse, sondern auch non-formal und informell erworbene Kompetenzen.

Die Gewerkschaften sollten sich intensiv mit der Umsetzung und Gestaltung der EU-Empfehlungen auf nationaler Ebene beschäftigen, um Reformen der Berufsbildung im Sinne der Beschäftigten zu befördern. Gelingt es, ein aussagekräftiges System der Validierung non-formal und informell erworbener Kompetenzen für den Bereich der beruflichen Weiterbildung zu entwickeln, kommt es darauf an, die sich daraus ergebenden Möglichkeiten in der betrieblichen Praxis zu verankern. Allerdings sollten Folgewirkungen des DQR für Arbeitnehmerinnen und Arbeitnehmer, deren Kompetenzentwicklung sowie Berufs- und Arbeitsbiografien für den Arbeitsmarkt und die Personal- und Organisationsentwicklung der Unternehmen untersucht werden. Chancen und Risiken müssen sichtbar gemacht werden.

\section{AUTOR}

HERMANN NEHLS, Diplom-Betriebswirt, ist Referatsleiter beim DGB-Bundesvorstand. Arbeitsschwerpunkte: Grundsatzfragen der Berufsbildungspolitik, berufliche Weiterbildung, europäische Berufsbildung.

hermann.nehls@dgb.de 